\title{
Measuring Gastrointestinal Electrical Activity With Extracellular Electrodes
}

TO THE EDITOR: We read with interest the paper by Worth et $\mathrm{al},{ }^{1}$ concerning the regulation of gastric electrical and mechanical activity by cholinesterases. We congratulate the authors on this interesting study.

However, we were surprised to read the following statements: "Recording electrical activity by extracellular array electrodes was thought in the past to be a more effective method for detecting breakdown in electrical continuity.... However, recordings via this technique are due largely to movement artifacts rather than to valid electrophysiological recordings of membrane currents (slow waves).”
The authors support this statement with a single reference, ignoring all competing evidence supporting the validity of extracellular recordings, as addressed in recent publications, editorials and letters. ${ }^{2-6}$ It is important to present a balanced factual assessment on the validity of extracellular recordings, so that readers and reviewers remain correctly informed about the technique.

In the study cited by Worth et al, ${ }^{1}$ slow waves could not be recorded in vitro using extracellular electrodes. However, failing to record slow waves in one study does not mean they cannot be recorded generally. The claims in this study have been discredited for well-documented reasons, ${ }^{2-5}$ including extensive use of in-

A Suction electrode signal

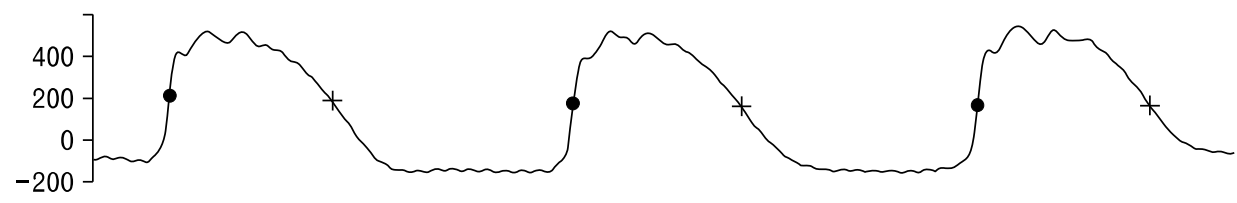

B Suction electrode signal - First derivative smoothed

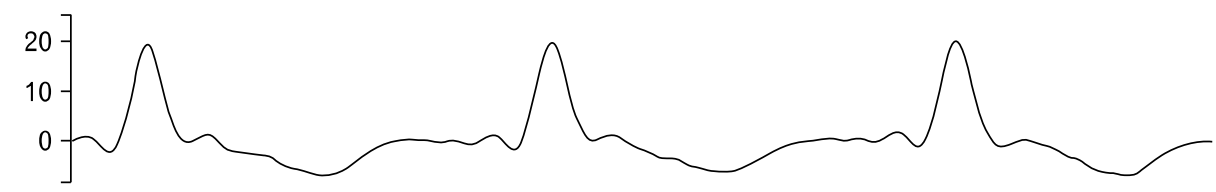

C Suction electrode signal - Second derivative smoothed

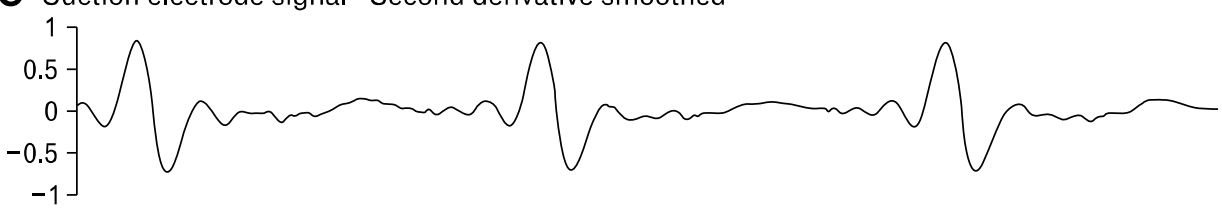

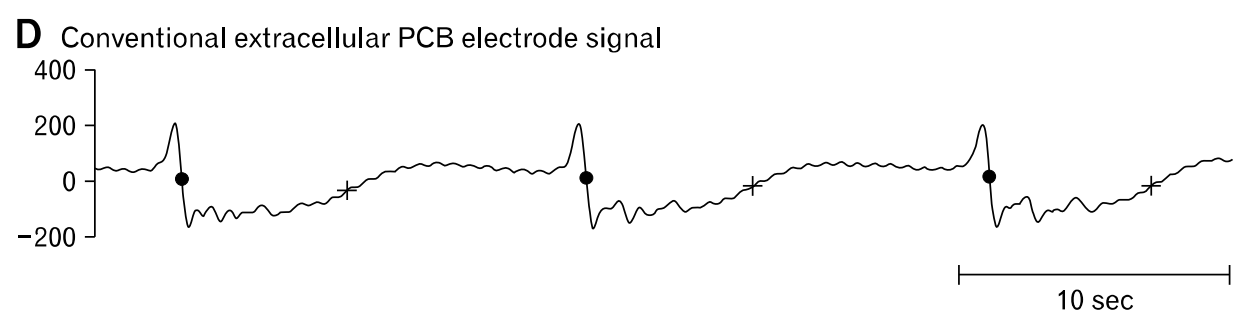

Figure. The morphology of suction extracellular slow wave potentials (A) approximates intracellular slow wave recordings, while their second derivative (C) appropriately approximates the morphology of potentials recorded by conventional contact extracellular electrodes (D). Adapted from Angeli et al. ${ }^{4}$ 
correct filters. ${ }^{6}$ In addition, extracellular recordings are more challenging in vitro, as coherent propagating wavefronts are required, and frequency gradients can be disturbed in isolated preparations. ${ }^{3}$

We invite the authors to consider the following data, from our recent in vivo study validating extracellular recordings (Figure), ${ }^{4}$ showing representative gastric slow wave recordings from multiple extracellular modalities, consistent with a century of extracellular studies. ${ }^{7}$

We invite the authors to consider: (1) how movement artifacts could generate 2 such different configurations across 2 extracellular methods (Figure A and D), which happen to ideally match slow wave membrane potential biophysics? ${ }^{4}$ And (2) how movement artifacts could explain such data given that tissue motion in our study was completely suppressed using nifidepine, as demonstrated in high-definition video mapping? $?^{4}$

Clearly, extracellular recordings accurately reflect slow wave membrane potential fields when correctly applied, and they therefore remain a "gold standard" tool in gastrointestinal physiology. ${ }^{8}$ In truth, extracellular array recordings would have been an ideal method for Worth et $\mathrm{al}^{1}$ to use, as they generate rich spatiotemporal data on slow wave propagation, ${ }^{9,10}$ which would have nicely complimented their excellent motility maps.

\section{Gregory $0^{\prime}$ Grady, ${ }^{1,2}$ Timothy R Angeli, ${ }^{2}$} Peng $\mathrm{Du}^{2}$ and Leo $\mathrm{K}$ Cheng ${ }^{2,3}$

${ }^{1}$ Department of Surgery, University of Auckland, Auckland, New Zealand;

${ }^{2}$ Auckland Bioengineering Institute, University of Auckland, Auckland, New Zealand; and ${ }^{3}$ Department of Surgery, Vanderbilt University, Nashville, Tennessee, USA
1. Worth AA, Forrest AS, Peri LE, Ward SM, Hennig GW, Sanders KM. Regulation of gastric electrical and mechanical activity by cholinesterases in mice. J Neurogastroenterol Motil 2015;21:200-216.

2. O'Grady G. Gastrointestinal extracellular electrical recordings: fact or artifact? Neurogastroenterol Motil 2012;24:1-6.

3. O'Grady G, Pullan AJ, Cheng LK. The analysis of human gastric pacemaker activity. J Physiol 2012;590(Pt 5):1299-1300.

4. Angeli TR, Du P, Paskaranandavadivel N, et al. The bioelectrical basis and validity of gastrointestinal extracellular slow wave recordings. J Physiol 2013;591(Pt 18):4567-4579.

5. O'Grady G, Angeli T, Du P, Cheng LK. Concerning the validity of gastrointestinal extracellular recordings. Physiol Rev 2015;95:691692.

6. Paskaranandavadivel N, O'Grady G, Du P, Cheng LK. Comparison of filtering methods for extracellular gastric slow wave recordings. Neurogastroenterol Motil 2013;25:79-83.

7. Szurszewski JH. A 100-year perspective on gastrointestinal motility. Am J Physiol 1998;274(3 Pt 1):G447-G453.

8. Sarna SK. The gold standard for interpretation of slow wave frequency in in vitro and in vivo recordings by extracellular electrodes. $\mathrm{J}$ Physiol 2013;591(Pt 18):4373-4374.

9. Lammers WJ, Stephen B, Karam SM. Functional reentry and circus movement arrhythmias in the small intestine of normal and diabetic rats. Am J Physiol Gastrointest Liver Physiol 2012;302:G684-G689.

10. O'Grady G, Angeli T, Du P, et al. Abnormal initiation and conduction of slow wave activity in gastroparesis, defined by high-resolution electrical mapping. Gastroenterology 2012;143:589-598, e1-e3.

\section{Conflicts of interest: None.}

